\title{
Profile of Hypertensive Retinopathy in a Tertiary Centre in Western Nepal
}

\author{
Pun $\mathrm{CB}^{1}$, Tuladhar $\mathbf{S}^{* 2}$ \\ ${ }^{1}$ Associate professor, Department of Medicine, ${ }^{2}$ Associate professor, Department of Ophthalmology, \\ Gandaki Medical College \& Teaching Hospital, Pokhara, Nepal
}

\section{Keywords}

Fundus, Hypertensive retinopathy,

Tertiary centre.

\section{Corresponding author}

${ }^{*}$ Dr. Sarita Tuladhar

Associate professor

Dept of Ophthalmology

Gandaki Medical College \& Teaching

Hospital Pokhara, Nepal

Email: drtuladharsarita@yahoo.com

\begin{abstract}
Objective: The present study was done to find out prevalence of hypertensive retinopathy among patients attending in OPD in GMC.

Methods: A hospital based cross-sectional study was performed among 200 hypertensive patients visiting eye OPD from Dec 2016 to Dec 2017. Detailed eye examination including fundus evaluation under mydriasis was done on all subjects and hypertensive retinopathy was graded according to Keith, Wagner and Barker classification by ophthalmologist using 90 dioptre lens. Patient having diabetes and other retinal diseases were excluded from the study. Data analysis was done using spss software.
\end{abstract}

Results: The mean age of the patients was $60.58 \pm 12.26$ standard deviation. In our study $56.5 \%$ patients had hypertensive retinopathy. Of which $31 \%$ had grade I, $19 \%$ had grade II, $6 \%$ had grade III and $0.5 \%$ had grade IV hypertensive retinopathy. The ratio of hypertensive retinopathy among male and female was 1.7:1.

Conclusion: Hypertensive retinopathy is commonamong hypertensives and males are more prone to retinopathy than females.

\section{INTRODUCTION}

Hypertension affects nearly $26 \%$ of the adult population worldwide. Kearney and colleagues estimated that the prevalence of hypertension in 2000 was $26 \%$ of the adult population globally and that in 2025 the prevalence would increase by $24 \%$ in developed countries and $80 \%$ in developing countries ${ }^{1}$.

Hypertension is a risk factor for a number of visionthreatening eye conditions including retinal vascular occlusion, retinal macroaneurysm and non arteritic anterior ischaemic optic neuropathy.

Eyes are proven hypertensive target organs ${ }^{2}$. Hypertension may exacerbate the vision-threatening effects of diabetic retinopathy and has been implicated in the pathogenesis of age-related macular degeneration. Ocular involvement in the setting of hypertension was described by Liehroich in 1859. Fundamentally, the effects of ocular changes arise from the impact of the ocular vasculature.

Hypertensive retinopathy ocular changes occur in the rational circulation in the acute stage of hypertension, primarily involving the terminal arteries rather than the main retinal arteries. The main retinal arteries changes are seen in the respond to chronic hypertension. Retinal microvascular changes are signs of hypertensive retinopathy and can be useful to classify risk factors and treatment decisions for hypertension ${ }^{3}$.

In general, the degree and duration of the hypertension are primarily determined on hypertensive retinopathy fundus changes. These retinopathy fundus changes may be seen in the other diseases with vascular risk factors such as diabetes. The retinopathy fundus changes may also be more severe and more progressive when diabetes and hypertensive are associated. Other factors such as hyperlipidemia may make the retinopathy worst as well. The subject of hypertensive retinopathy fundus changes 
is still the focus of many controversies. The optic disc edema and retinal pigment epithelial tissue represent manifestation of hypertensive optic neuropathy.

Earlier detection of hypertensive patients who are in risk to develop retinopathy is very important. So, this study performed to estimate the prevalence of hypertensive retinopathy.

\section{METHODS}

A hospital based cross-sectional study was performed among 200 hypertensive patients visiting eye OPD from Dec 2016 to Dec 2017. Patients having blood pressure higher than normal $(>140 / 90 \mathrm{~mm}$ of $\mathrm{Hg}$ ) were included in the study. Most of them were already diagnosed case of hypertension on medication but not well controlled and some were newly diagnosed.

All hypertensive patients were enquired about the duration of hypertension and treatment. Most patients had uncontrolled blood pressure. A detailed examination of the eye was carried out including fundus examination under mydriasis with direct ophthalmoscope, +90 D lens and Goldman 3 mirror lens where necessary.

\section{RESULTS}

The mean age of the patients in our study was $60.58 \pm 12.26$ years. Table 1 shows the age distribution of patients in different age groups.

Table 1: Age distribution of patients

\begin{tabular}{lcc}
\hline Age group (Years) & No. of patients & Percentage \\
$30-40$ & 24 & $12 \%$ \\
$41-50$ & 36 & $18 \%$ \\
$51-60$ & 53 & $26.5 \%$ \\
$61-70$ & 45 & $22.5 \%$ \\
$71-80$ & 32 & $16 \%$ \\
$>80$ & 10 & $5 \%$ \\
Total & 200 & $100 \%$ \\
\hline
\end{tabular}

In our study 87 patients i.e $43.5 \%$ were hypertensive without retinopathy changes and 113 patients i.e. 56.5\% were hypertensives with retinopathy fundus changes. Table 2 shows grading of hypertensive retinopathy according to Keith-Wagner Barker grading in different sexes.
Table 2: Grading of hypertensive retinopathy

\begin{tabular}{lcccc}
\hline Keith Wagener & Males & Females & Total & Percentage \\
Grade I & 38 & 24 & 62 & $31 \%$ \\
Grade I & 26 & 12 & 38 & $19 \%$ \\
Grade III & 6 & 6 & 12 & $6 \%$ \\
Grade IV & 1 & 0 & 1 & $0.5 \%$ \\
Total & 71 & 42 & 113 & $56.5 \%$ \\
\hline
\end{tabular}

In our study 71 patients were males and 42 were females. So the male: female ratio was 1.7 .

\section{DISCUSSION}

Systemic hypertension is a chronic multi-factorial disease involving brain, heart, eyes, and kidneys. Systemic hypertension affects arteries, veins, choroid and optic nerve in eyes.

In our study more males were affected than females. Similar results were seen in a study by Mondal RN in Bangladesh ${ }^{4}$ while studies showed more females than males ${ }^{5}$.

The mean age of the patients in our study was $60.58 \pm 12.26$ years. Similar age group was seen in studies by Bastola $\mathrm{P}$ et $a l^{6}$. Other studies Mondal $\mathrm{RN}^{4}$ showed hypertension retinopathy at fifties.

In our study, $56.5 \%$ of the hypertensive patients had retinopathy changes and $43.5 \%$ patients without retinopathy fundus changes. Similar finding was seen in a study by Erden S, Bicakci E which showed hypertensive retinopathy in $66.3 \%{ }^{7}$. But studies by Kabedi et al ${ }^{8}$ stated hypertensive retinopathy incidence of $83.6 \%$.Some studies ${ }^{4}$ showed a lower rate too (29.9\%). Likewise study by Klein $\mathrm{R}$ et $a l^{9}$ showed lower rate of hypertensive retinopathy as in $7.8 \%$. The higher prevalence of hypertensive retinopathy in our study may be due to late presentation of the patients to the hospital, uncontrolled hypertension, patients not taking medicines regularly due to lack of awareness.

In our study, prevalence of grade I and II hypertensive retinopathy was $38 \%$ and $26 \%$ respectively. Only $6 \%$ had grade III hypertension retinopathy. As per the study conducted by Del Brutto et $a l^{10}$, hypertensive retinopathy grade 1 was recorded in $37 \%$, and grade 2 hypertensive retinopathy was noted in $17 \%$ of hypertensive patients. Similar results showing more cases of grade I then II and few cases of grade III and IV were seen in other studies too $^{4,11,12}$. 


\section{CONCLUSION}

In our study $55.6 \%$ of hypertensive patients had retinopathy. In a country like Nepal where education and economy is poor, such studies help the population to be aware of the diseases and possible complications.

\section{REFERENCES}

1. Kearney PM, Whelton M, Reynolds K, et al. Global burden of hypertension: Analysis of worldwide data. Lancet. 2005; 365: 217-223.

2. Pache $\mathrm{M}$, Kube $\mathrm{T}$, Wolf S, et al. Do angiographic data support a detailed classification of hypertensive fundus changes? J Hum Hypertension. 2002; 16: 405-410.

3. Duncan BB, Wong TU, Tyroler HA, et al. Hypertensive retinopathy and incident coronary heart disease in high-risk men. Br J Ophthalmol. 2002; 86: 1002-1006.

4. Mondal RN, Matin MA, Rani M, et al. Prevalence and risk factors of hypertensive retinopathy in hypertensive patients. J Hypertens. 2017; 6: 241. doi:10.4172/2167-1095.1000241

5. Badhu BP, Shrestha JK. Hypertensive patients in eye OPD, TUTH. JIOM.1998; 20(3,4).

6. Bastola P, Pun CB, Koirala S, et al. Fasting serum lipids and fundus changes in hypertensive patients. NJMS. 2012; 1: 103-107.

7. Erden S, Bicakci E. Hypertensive retinopathy: Incidence, risk factors, and comorbidities. Clin Exp Hypertens. 2012; 34(6): 397-401.

8. Kabedi NN, Mwanza JC, Lepira FB, et al. Hypertensive retinopathy and its association with cardiovascular, renal and cerebrovascular morbidity in Congolese patients. Cardiovasc J Afr. 2014 Sep-Oct; 25(5): 228-32.

9. Klein R (1), Klein BE, Moss SE, et al. Hypertension and retinopathy, arteriolar narrowing, and arteriovenous nicking in a population. Arch Ophthalmology. 1994; 112: 92-8.
10. Del Brutto OH, Mera RM, Viteri EM, et al. Hypertensive retinopathy and cerebral small vessel disease in Amerindians living in rural Ecuador: The Atahualpa Project. Int J Cardiol. 2016 Sep 01; 218: 65-68.

11. Cuspidi C, Macca G, Sampieri L, et al. High prevalence of cardiac and extracardiac target organ damage in refractory hypertension. $J$ Hypertens. 2001; 19: 2063-2070. 